De los Reyes Lozano, J. (2015). Genética del doblaje cinematográfico. La versión del traductor como proto-texto en el filme Rio. Linguistica Antverpiensia, New Series: Themes in Translation Studies, 14, 149-167.

\title{
Genética del doblaje cinematográfico. La versión del traductor como proto-texto en el filme Rio
}

\author{
Julio de los Reyes Lozano
}

Université de Lille III, France \& Universitat Jaume I, Spain

julio.delosreyeslozano@univ-lille3.fr

This paper analyses the Spanish dubbed version of the animated film 'Rio' from a genetic point of view. Dubbing is the audiovisual translation mode which consists in re-recording the original voice track in the target language: although involving a large group of professionals, the translator's task is pivotal to this process as he or she is responsible for transferring the information from one language to another. However, the text produced by the translator is not what will be heard on screen, as it will go through some transformations and rewritings during the dubbing process. It might even be considered a work-in-progress text, since a closer look reveals a high presence of hesitations, comments and other remarks addressed to the next agents. Once detected and classified, these marks will help us describe the dialogic relationship among some of the professionals involved in the dubbing process.

\section{La traducción para el doblaje}

El doblaje es la modalidad de traducción audiovisual (TAV) que consiste en la sustitución, en la banda sonora de un texto audiovisual, de la pista de diálogos original por una nueva pista con la versión grabada en lengua meta. Su uso difiere de una zona geográfica a otra y, mientras en algunos países como España se trata de la principal modalidad de TAV, en otros como Portugal se prefiere la subtitulación. Con todo, algunos productos, como los filmes para niños, se doblan prácticamente en todo el mundo (Chaume, 2012, p. 6), como sucede con el corpus elegido en este trabajo: la película de animación Rio (Anderson, Donkin \& Saldanha, 2011).

El empleo de textos audiovisuales, cuya especificidad reside en que transmiten información de forma simultánea a través de dos canales de comunicación -acústico y visual- y diferentes códigos semióticos (Chaume, 2004, p. 15), confiere unas características especiales a la traducción para doblaje: entre ellas, destacan el uso prefabricado del lenguaje y la necesidad de reconocer la información ofrecida por los distintos códigos de significación (Chaume, 2004, p. 168). Por otro lado, el proceso de trabajo no se limita a la traducción de un texto por una persona, sino que requiere la intervención de varios profesionales. Sólo 
en España, "el texto audiovisual pasa por las manos de productor, distribuidor, exhibidor, director del estudio de doblaje (para casting y producción), traductor, adaptador, actores, director y técnicos de doblaje antes de llegar al emisor final" (Marzà, Torralba y Grupo TRAMA, 2013, p. 36). En algunas cadenas de televisión regionales también se incluye la figura del experto lingüista, quien comprueba la exactitud lingüística del texto y su pronunciación (Matamala, 2010, p. 103).

Si bien el eslabón principal de esta cadena lo constituye el traductor, quien conoce las dos lenguas de trabajo y permite en última instancia que se produzca la comunicación (Chaume, 2004, p. 68), el texto realizado por este agente es un texto "inacabado", un "proto-texto" que presenta varias soluciones lingüísticas e incluye comentarios, avisos y explicaciones y cuyo destinatario es el siguiente agente en la cadena de doblaje, el ajustador (Richart, 2009, p. 150) ${ }^{1}$. Este último, o en su defecto el ayudante de dirección, reescribe posteriormente el texto, dividiéndolo en takes y modificándolo para facilitar la correcta sincronización de los diálogos. A continuación, el texto ajustado pasa a la sala de grabación donde el director y los actores de doblaje aportan nuevos cambios teniendo en cuenta aspectos como el tono y el timbre de voz (Ávila, 1997, pp. 99-100). Por último, el cliente (o un supervisor designado por éste) comprueba el resultado final y, si procede, solicita las modificaciones necesarias (Matamala, 2010, p. 102).

La diversidad de agentes impide, por tanto, identificar a un único autor del doblaje, pues el mismo borrador pasa por varias manos antes de convertirse en definitivo ${ }^{2}$. Este borrador es "la «huella» de la obra, huella deformante y fugitiva" (Richart, 2009, p. 147) que documenta lo que ocurre en cada etapa y que conviene analizar desde la óptica genética, de forma fragmentada y cronológica si se quiere hallar al responsable último de cada modificación. El estudio que aquí planteo se acerca a la génesis del doblaje español de Rio partiendo del análisis del guión elaborado por el traductor. La inestabilidad que se percibe en el estadio creativo más inicial ayuda a reconocer el doblaje como una actividad colaborativa que, más que una traducción, es "un moldeamiento y transformación que supone un importante nivel de reescritura del texto de partida" (Richart, 2009, p. 184).

\section{El análisis genético del doblaje}

La investigación sobre TAV ha crecido de forma exponencial en la última década hasta el punto de haberse convertido en los últimos años en una de las locomotoras de los Estudios de Traducción (Chaume, 2013, p. 289). En su breve pero intensa historia, los investigadores se han centrado fundamentalmente en el análisis del proceso de traducción y del producto final estableciendo marcos metodológicos propios para distinguir las estrategias y técnicas de traducción, la labor de los profesionales 
implicados o las restricciones del medio audiovisual, entre otras cuestiones $^{3}$.

Aun enfatizando su carácter colaborativo, los estudios que exploran el ámbito profesional de la TAV suelen limitarse a detallar la cadena de trabajo y las diferentes etapas que la conforman, y son pocos los que analizan las modificaciones y manipulaciones que sufre el texto en cada fase, desde que se encarga su traducción hasta que se proyecta en la sala de cine. La ausencia de esta reflexión impide identificar de forma certera al agente responsable de las decisiones de traducción que, como hemos visto más arriba, no siempre es el traductor: ajustadores, directores o actores de doblaje también modifican el texto en función de las necesidades de cada etapa y, en ocasiones, las soluciones de traducción pueden provenir incluso de las propias productoras ${ }^{4}$. El análisis genético de la TAV comienza, por tanto, reconociendo su pluralidad de autores, al igual que el estudio de las manifestaciones artísticas que son fruto de la colaboración colectiva, como el cine y el teatro (Anokhina, 2010, p. 209).

Antes de la revolución digital, las modificaciones encontraban su reflejo documental en forma de tachones, reescrituras, borrones $y$ anotaciones en los borradores elaborados por cada agente (Richart, 2013, epígrafe 9.1). Hoy en día, gracias a la función de seguimiento y control de cambios de la mayor parte de editores de texto informáticos, es posible revisar, añadir comentarios y modificar el texto directamente en el archivo recibido, y a continuación reenviarlo "limpio" a través de Internet. Los borrones y tachones han sido sustituidos por otro tipo de marcas, que abordaré en el epígrafe dedicado al análisis del corpus. De cualquier modo, manuscritas o no, sólo es posible verificar las alteraciones realizadas en cada etapa si se dispone de la versión del archivo efectuada por cada agente.

Estas versiones conforman el archivo documental del doblaje que, como en un proceso traductológico unipersonal, ofrece información específica sobre la toma de decisiones en los diferentes estadios del proceso de traducción (Munday, 2013, p. 134). Su recopilación y análisis equivale en cierto sentido a la composición del avant-texte literario, es decir el conjunto de borradores, escritos, pruebas y variantes que preceden a un trabajo publicado previamente (Bellemin-Noël, 1972, p. 15). Para obtenerlo, el investigador debe recopilar, clasificar, fechar y descifrar todos los documentos del expediente genético de la obra con el fin de otorgarle un significado lúcido. La sucesión cronológica de las operaciones que desembocan en el texto final permite arrojar luz sobre los mecanismos de creación del autor (de Biasi, 2011, pp. 68-70).

Sin embargo, la construcción del avant-texte para el estudio de la TAV no siempre es sencilla ni factible, pues supone seguir la huella de textos inéditos realizados por personas distintas, cuya conservación y publicación dependen de la voluntad de cada agente. El simple hecho de tener que solicitar estos documentos a más de una persona complica sobremanera la tarea del investigador y representa un grave obstáculo 
para el avance del estudio genético. Richart (2009, p. 166) retoma la noción de Derrida (1995) y denuncia el "mal de archivo" existente en la investigación sobre doblaje. A éste contribuyen especialmente las propias empresas de doblaje, que guardan celosamente su documentación y ponen todo tipo de impedimentos para que el investigador acceda a ella, obligando a los responsables a firmar acuerdos de confidencialidad para proteger los derechos de autor. Por otra parte, no siempre queda constancia de la documentación, pues "el departamento técnico realiza copias de trabajo y seguridad que serán destruidas al finalizar el proceso" (Ávila, 1997, p. 33).

En consecuencia, los trabajos con base genética son casi inexistentes en TAV y los pocos que se aproximan a ella lo hacen desde una perspectiva histórica, buscando desvelar las razones artísticas, técnicas y socio-económicas que motivaron su advenimiento (di Carlo, 1995; Cornu, 2014). Hasta la fecha, sólo se tiene constancia de dos investigaciones prácticas: una de ellas es la de Matamala (2010), quien define la traducción para el doblaje como un conjunto de textos dinámicos que cambian a lo largo del proceso.

Este artículo describe los cambios que se producen en el guión traducido durante las fases de sincronización, revisión lingüística y grabación de diálogos en los doblajes catalán y español de tres películas de acción recientes. La autora constata que la mayor parte de modificaciones se realiza en la etapa de sincronización y que las estrategias de traducción más empleadas a tal fin son la reducción y la amplificación (Matamala, 2010, p. 113). En cuanto a la comparación entre lenguas meta, mientras que en español predominan las alteraciones en la etapa de sincronización, los cambios en la versión catalana se concentran en la fase de revisión lingüística.

Con un enfoque más teórico, Richart $(2009,2013)$ presenta un modelo de análisis que tiene por objeto recopilar e interpretar la documentación creada por los distintos agentes implicados en el proceso. Éste se divide en cuatro etapas bien diferenciadas (Richart, 2013, cap. 9): en primer lugar se hallan los textos de partida, donde se incluyen tanto el texto audiovisual, el guión para el doblaje y la lista de diálogos como material anterior en los que se inspira el producto audiovisual, así como las explicaciones y recomendaciones realizadas por el emisor; en segundo lugar, aparece el texto producido por el traductor, un primer borrador en lengua meta que Richart denomina "proto-texto" o "proto-traducción" y que contiene comentarios y propuestas dirigidas al siguiente agente, el ajustador; en tercer lugar, el texto del ajuste contiene las modificaciones efectuadas por el ajustador sobre el texto anterior, que responden fundamentalmente a la influencia de la imagen en el texto audiovisual; en cuarto y último lugar, antes de llegar a las pantallas, el texto también sufre alteraciones en la sala de doblaje, por parte principalmente del director y los actores de doblaje. 
La tarea del investigador consiste, por tanto, en fragmentar el análisis del texto audiovisual en torno a estas cuatro etapas, examinar qué sucede en cada una de ellas y describir el conjunto de transformaciones que se llevan a cabo hasta obtener el resultado final: el texto doblado que oímos en el cine. Además, este modelo de análisis es valorativo, pues trata de interpretar el porqué de estas manipulaciones, y a qué o a quién benefician desde un punto de vista ideológico (Richart, 2013, epígrafe 9.5). El interés para los investigadores en traducción es pues doble: por un lado, demuestra de forma empírica la complejidad y el carácter dialógico del proceso de trabajo; y por otro, permite revelar los motivos que llevan a los distintos agentes a modificar el texto.

\section{Metodología y corpus}

El estudio que aquí presento sigue el marco establecido por Richart y centra su mirada principalmente en el texto elaborado por el traductor para el doblaje de Rio, por ser éste el eslabón principal de la cadena de trabajo. Para ello, se describen los mecanismos de diálogo que emplea el traductor para dirigirse al ajustador. Las notas, dudas, propuestas múltiples y comentarios convierten al texto del traductor en un borrador sin terminar, un "proto-texto" sujeto a modificaciones en las fases sucesivas. El análisis estadístico y la clasificación de dichas señales constituyen, por tanto, la primera parte del estudio. La consulta de los documentos posteriores (texto del ajuste y doblaje definitivo) permitirá seguir el recorrido del texto de trabajo y comprobar el margen de modificación existente en la cadena de doblaje.

El filme Rio es un largometraje de animación por ordenador producido por Blue Sky Studios y dirigido en 2011 por el realizador brasileño Carlos Saldanha. Cuenta la historia de Blu, un guacamayo azul en peligro de extinción que lleva una cómoda vida en Minnesota junto a su dueña, Linda. Su rutina se verá alterada cuando Tulio, un ornitólogo brasileño, los lleva a Río de Janeiro para que conozcan a Perla, una hembra de guacamayo que podría salvar la especie. En su viaje a Brasil, Blu recuperará su instinto natural, olvidado después de varios años de vida doméstica y, tras enfrentarse a una banda de contrabandistas de animales, aprenderá a valorar la importancia de la libertad para las aves y la necesidad de proteger su hábitat.

Al igual que la mayoría del cine para niños actual, esta película se dirige a un doble receptor, infantil y adulto, formando parte de lo que se conoce por "textos ambivalentes" (Shavit, 1980, p. 76). En ellos, se relata una historia de fácil comprensión para el niño, salpicada de elementos concebidos para el disfrute adulto, tales como referencias culturales e intertextuales, dobles sentidos o juegos de palabras. Al trasladarse a otra cultura, estos elementos no siempre aparecen en la versión doblada, dando lugar a textos más univalentes que los originales (O'Connell, 2003, 
p. 227). Para ello, los agentes suelen intervenir con cierta libertad en las adaptaciones lingüísticas de estos productos, tanto en la traducción como en el estudio de doblaje, simplificándolos, familiarizándolos, o simplemente modificándolos (Ruzicka, 2009, p. 8).

En este sentido, resulta interesante comprobar las decisiones del traductor y los agentes posteriores ante elementos considerados como problemáticos para el niño, bien por su dificultad de comprensión, bien por su carácter inadecuado. Y también es útil ver hasta qué punto influye la ideología de la sobreprotección en la traducción de lo que ve y escucha el niño de la cultura meta. En esta investigación, se identifican los siguientes objetivos:

- En primer lugar, identificar las marcas, en el texto del traductor, que se dirigen a los siguientes agentes de la cadena del doblaje con el fin de demostrar el carácter vacilante e inacabado del documento.

- En segundo lugar, clasificar estas marcas en función de su función y significado.

- En tercer lugar, comprobar si dichas marcas encuentran su reflejo en el texto del ajuste y en el doblaje definitivo; y distinguir los motivos que llevan a adoptar o rechazar la opción del traductor.

En consecuencia, se formulan las siguientes hipótesis de trabajo:

- Hipótesis 1: el texto del traductor contiene reflexiones, vacilaciones, comentarios y recomendaciones que lo convierten en un "proto-texto" o "proto-traducción". Estas marcas podrán recogerse o rechazarse en las sucesivas etapas del doblaje.

- Hipótesis 2: la decisión de tener en cuenta o no la información adicional proporcionada por el traductor se debe a razones ideológicas o a restricciones propias del texto audiovisual: dado que Rio es un filme concebido para la audiencia familiar, algunos elementos tratarán de adecuarse al espectador infantil. Asimismo, los diferentes códigos de significación que construyen el texto también influyen en su traducción.

La primera parte de la investigación requirió la puesta en contacto con los responsables de la adaptación al español para obtener los documentos de trabajo. Si bien el estudio de doblaje y el traductor suelen aparecer al final de los títulos de crédito en muchos filmes, no sucede así en la edición española de Rio en DVD, que es la que aquí se utiliza para el análisis del

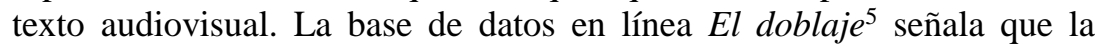
adaptación corrió a cargo del ya extinto estudio barcelonés Sonoblok, quien a su vez confió en Josep Llurba como traductor y en Gonzalo Abril como ajustador y director de doblaje. Tras escribirles, éstos proporcionaron desinteresadamente una copia electrónica de sus 
respectivos borradores (el guión traducido y el texto ajustado y pautado) que, junto a las versiones original y doblada del texto audiovisual, conforman el archivo documental de la presente investigación ${ }^{6}$.

\section{El texto del traductor en el filme Rio}

El análisis del texto del traductor se llevó a cabo en dos fases, cuantitativa y cualitativa, en busca de marcas dirigidas a los agentes posteriores. Las cifras arrojan un resultado de 1097 recurrencias (Tabla 1), que se han agrupado en cinco categorías ${ }^{7}$ : notas del traductor, utilizadas principalmente para comentar problemas relacionados con el texto original; propuestas múltiples que ofrecen dos e incluso tres soluciones de traducción; amplificaciones sobre las decisiones de traducción; dudas sobre la comprensión del texto de partida; por último, los símbolos del doblaje que describen información paralingüística.

Tabla 1. Indicaciones efectuadas en el texto del traductor

\begin{tabular}{|l|l|}
\hline Tipo de indicación & Cantidad \\
\hline Notas del traductor & 21 \\
\hline Propuestas multiples & 84 \\
\hline Amplificaciones & 47 \\
\hline Dudas & 3 \\
\hline Símbolos del doblaje & 942 \\
\hline TOTAL & 1097 \\
\hline
\end{tabular}

El análisis cualitativo describe la función de las marcas encontradas y las coteja con los textos sucesivos, el texto del ajuste (TA) y el texto definitivo (TD), con el fin de comprobar su grado de relevancia en el proceso de producción. Las siguientes líneas son un resumen del mismo, que se ilustra con ejemplos específicos de cada parámetro y en el que se pone de manifiesto el continuo estado de revisión al que está sometido el texto del traductor.

\subsection{Notas del traductor}

Es quizá el mecanismo más visible que utiliza el traductor para dirigirse a los agentes posteriores. En este texto, las notas del traductor siempre van precedidas de la mención "*NOTA DEL T" y su función es explicar y justificar las soluciones adoptadas ante elementos del texto original que 
pueden suponer un problema de traducción, tales como dobles sentidos, unidades fraseológicas, referencias culturales e intertextuales o la confluencia entre los códigos lingüístico e iconográfico.

Tabla 2. Notas del traductor: Función y empleo en el texto definitivo (TD)

\begin{tabular}{|l|l|l|l|}
\hline Notas del traductor & Cantidad & TD & No TD \\
\hline Dobles sentidos lingüísticos & 6 & 4 & 2 \\
\hline Unidades fraseológicas & 5 & 1 & 4 \\
\hline Confluencia de códigos & 4 & 2 & 2 \\
\hline Referencias culturales e intertextuales & 4 & 3 & 1 \\
\hline Otras & 2 & 2 & 0 \\
\hline TOTAL & 21 & 12 & 9 \\
\hline
\end{tabular}

Según la Tabla 2, de las 21 notas existentes en el texto, en 12 ocasiones se mantiene la opción explicada y justificada por el traductor en el TD (57\%), mientras que en 9 casos se opta por una solución diferente (43\%). Los motivos de esta última opción se entremezclan, aunque la ideología (5 veces) y las restricciones de la imagen (4 veces) suelen predominar.

El siguiente ejemplo representa el principal reto al que se enfrenta el traductor de productos audiovisuales, la interacción de los códigos lingüístico e iconográfico. De forma transversal, se introduce una referencia cultural y la confluencia de los factores da pie al humor audiovisual. En él, asistimos al primer encuentro entre los pájaros protagonistas y Blu explica el origen de su nombre ${ }^{8}$ (el subrayado es mío):

(1) Hi. My name is Blu. You know like the cheese with the mold on it. No, it smells really bad. That's stupid, stupid, stupid.

Hola. Me llamo Blu. Ya sabes, como el color azul. El de ese queso que lleva moho. Y que huele muy mal. Qué estupidez. Estúpido, estúpido, estúpido.

*NOTA DEL T: En el original dice directamente que se llama "Blu" como el queso ("blue cheese" = queso azul). En castellano he añadido "como el color azul".

El chiste se construye a partir del nombre propio y su alusión al queso azul: que las plumas sean de color azul es la razón de que el ave se llame "Blu", que se pronuncia igual a la palabra blue (azul) y que le lleva a compararse con el queso homónimo. La opción de no traducir el nombre del pájaro sobrepasa el poder de decisión del traductor ${ }^{9} \mathrm{y}$, además, se 
aparta de la tendencia usual en el cine para niños, donde se suelen traducir los antropónimos que tienen algún significado relacionado con la trama o el código iconográfico (Borràs y Matamala, 2009, p. 291). De este modo, el traductor se ve obligado a mencionar el color del pájaro, pues el código lingüístico se encuentra subordinado al iconográfico. Para ello, explicita el significado de "Blu" y justifica su propuesta detallando en la nota el sentido del texto original.

Veamos lo que sucede en los pasos sucesivos (la cursiva y el subrayado son míos):

TA: (G) Hola. Me llamo Blu. (S) Como el color azul. / Y como ese queso/ que tiene moho. (DE) Y huele mal.

TD: (G) Hola. Me llamo Blu. (S) Como ese queso/ que tiene moho.

(DE) Y huele mal.

Además de incluir los símbolos paralingüísticos destinados al director y a los actores de doblaje, el TA modifica ligeramente la frase, sintetizando al máximo la oración pero manteniendo la idea principal del traductor para explicar el significado de "Blu". Sin embargo, dicha solución no se utilizará finalmente en el TD donde se suprime por completo la frase "como el color azul" para garantizar la isocronía ${ }^{10}$. Esta ausencia impide al espectador enlazar una y otra referencia, lo que puede llevarle a salir momentáneamente de la historia.

Para evitarlo, y atendiendo a todas las restricciones, se podría especificar el nombre español del queso con la siguiente frase: "Como el queso azul, que tiene moho y huele mal". O bien modificar el enunciado por completo: nada impide suprimir la alusión al queso, pues no existe ninguna referencia visual a este alimento que obligue a mantenerla, y sustituirla por otro elemento que sea azul y desagradable a la vez. De hecho, aunque no reproduzca el mismo chiste, el texto meta puede introducir otro diferente que consiga igualmente provocar una sonrisa -tal vez mayor- en la audiencia (Martínez Sierra, 2009, p. 194).

Veamos otro caso que también fue modificado en el TD, cuyo mayor reto traductológico es una unidad fraseológica. Nigel, una cacatúa acosadora y matona captura a Perla y la mantiene agarrada del cuello, impidiéndole hablar:

(2) Hello, pretty bird. What's the matter? Cockatoo got your throat?

Hola, pichoncita. ¿Qué te pasa? ¿Estás de la cacatúa hasta el cuello?

*NOTA DEL T: En el original, Nigel juega con la expresión "Cat got your tongue" (¿Se te comió la lengua el gato?), cuando dice "Cockatoo got your throat?” (¿La cacatúa se te comió (te ha atrapado) la garganta?) 
El código iconográfico vuelve a ser determinante en la propuesta del traductor, donde se priorizan las alusiones a la cacatúa y al cuello que aparecen en la imagen. A continuación, la nota explica el doble sentido de la frase original y la referencia a la popular expresión "cat got your tongue" y propone alternativas a su solución. A diferencia del ejemplo anterior, el TA se limita aquí a señalar los símbolos del doblaje, y es en la etapa final donde se transforma la expresión (la cursiva y el subrayado son míos):

TA: (OFF) Hola,/(ON) pichoncita./ ¿Qué te pasa? ¿Estás de la cacatúa hasta el cuello?

TD: (OFF) Hola,/(ON) pichoncita./ ¿Qué te pasa? ¿Te ha comido la lengua el loro?

Aunque es difícil dar con las razones exactas, en esta modificación se puede intuir una cuestión ideológica. La opción del TD se acerca más al equivalente acuñado de la frase original (ite ha comido la lengua el gato?) cambiando al gato por el loro, pero no consigue reproducir la idea de que es la cacatúa quien la mantiene asida de la garganta. Por su parte, la opción del traductor sí transmite estas referencias visuales, si bien para ello emplea una expresión coloquial quizá demasiado dramática para el público infantil, "estar hasta el cuello" (estar en gran aprieto). Así, la manipulación conlleva en este caso una dulcificación del texto meta pensando en el destinatario infantil, como ocurre en la traducción de otros textos para niños (Ruzicka, 2009).

Las siguientes muestras de justificación y explicación a través de las notas del traductor sí aparecen tanto en el TA como en el TD. En el ejemplo 3, el traductor argumenta el cambio de tratamiento -de formal a informal- entre los dos personajes humanos, mientras que en el ejemplo 4 argumenta su traducción de la conocida referencia intertextual a Terminator 2: el juicio final (Cameron, 1991):

(3) *NOTA DEL T: Aunque ya se llamaban por el nombre de pila, estos dos personajes se hablaban de usted. He pensado que este podría ser un buen momento para que empiecen a tutearse. Si no, no vuelve a haber un momento como este (de encandilamiento) hasta la bobina 4 .

(4) *NOTA DEL T: Aquí dice "Hasta la vista" en español. He añadido la opción que en "Terminator 2" se usó para "Hasta la vista, baby" (Sayonara, baby).

La importancia de las notas del traductor en este texto va más allá de la simple comunicación entre los agentes de la cadena de doblaje, pues gracias a ellas, el traductor se anticipa y defiende por adelantado sus propuestas, minimizando retrasos en el proyecto por causas lingüísticas. 


\subsection{Propuestas múltiples}

Otro de los elementos que muestran el carácter inacabado de la traducción es su tendencia a ofrecer más de una posibilidad de traducción para una situación concreta, dando lugar a un "texto-lexicón" (Richart, 2013, epígrafe 9.6.2) que permite a los siguientes agentes elegir dependiendo de la restricción a la que se enfrenten. En este borrador se cuentan hasta 84 propuestas múltiples de traducción -82 dobles y 2 triples-, que el traductor emplaza inmediatamente después de la solución que podemos calificar como principal, entre paréntesis y precedidas del símbolo $=$, como en los siguientes ejemplos (el subrayado es mío):

(5) Yeah, baby! Now I could get my freak on! ¡Sí, nena! ¡Ahora ya puedo soltarme el pelo (=bailar con desenfreno)!

(6) Okay, hold it together. The key is not to panic.

Bueno, cálmate un poco. La clave es no dejarse llevar por el pánico (=la clave es no ponerse nervioso).

(7) Because I'm still on Minnesota's time.

Eh, porque todavía estoy con el horario de Minnesota (=porque todavía tengo un poco de "jet lag")(desfase horario).

TA: Aún sigo con el horario de Minnesota.

TD: Ya sabes, cosas del "jet lag".

La diversidad de elección da su fruto y el TD opta en el 56\% de los casos por una de las opciones propuestas por el traductor: en 22 ocasiones (26\%) la solución principal (ejemplo 5) y en 25 ocasiones $(30 \%)$ por la opción u opciones adicionales (ejemplo 6). Sin embargo, el $44 \%$ restante no llegó al TD, primando el TA hasta en 28 ocasiones (33\%) frente a 9 nuevas propuestas (11\%) en la última fase (ejemplo 7). Como se observa en la Tabla 3, el respeto de la isocronía y de la sincronía labial son los principales motivos de exclusión de las soluciones del traductor. 
Tabla 3. Propuestas múltiples rechazadas: Tipo de restricción y soluciones adoptadas en el ajuste (TA) y el texto definitivo (TD)

\begin{tabular}{|l|l|l|l|}
\hline Tipo de restricción & Cantidad & TA & TD \\
\hline Isocronía & 13 & 9 & 4 \\
\hline Sincronía labial & 10 & 8 & 2 \\
\hline Sincronía cinética & 2 & 2 & 0 \\
\hline Código iconográfico & 1 & 0 & 1 \\
\hline Ideología & 1 & 1 & 0 \\
\hline Ninguna & 10 & 8 & 2 \\
\hline TOTAL & 37 & 28 & 9 \\
\hline
\end{tabular}

\subsection{Amplificaciones}

De manera similar a las propuestas múltiples, el traductor también ofrece opciones alternativas mediante posibles amplificaciones a una misma solución. Para ello, incluye entre paréntesis pequeñas explicitaciones o precisiones que no aparecen en el texto original. Como se observa en la Tabla 4, estas amplificaciones se utilizan para señalar el multilingüismo (28 veces), para explicitar los gestos paralingüísticos (13 veces) y para proponer una ligera variación a la solución de traducción (6 veces):

Tabla 4. Amplificaciones: Función y cantidad

\begin{tabular}{|l|l|}
\hline Amplificaciones & Cantidad \\
\hline Multilingüismo & 28 \\
\hline Explicitación de gestos & 13 \\
\hline Explicitación lingüística & 6 \\
\hline TOTAL & 47 \\
\hline
\end{tabular}

En el último caso, la amplificación entre paréntesis le permite a los agentes sucesivos optar por un parlamento más o menos extenso en función de la imagen: si, por ejemplo, la boca del personaje permanece abierta en pantalla durante mucho tiempo, el ajustador o el director de doblaje podrán optar por la frase más larga y viceversa. Del mismo modo, una escena de acción, en la que lo importante es el movimiento y la voz queda en un segundo plano, puede requerir el uso de frases más cortas. 
De las 6 muestras de este tipo de amplificación en el texto, el TD no hizo uso de ninguna, como en los siguientes ejemplos (el subrayado es mío):

(8) Hey, hey don't talk to me about nature. I watch Animal Planet.

Eh, eh, a mí no me hables de naturaleza, que veo (los documentales de) "Animal Planet".

(9) Wait, stop please. Performers only.

Un momento. Alto, por favor. Solamente participantes (en el desfile).

Las amplificaciones también se emplean para explicitar los gestos paralingüísticos (13 ocasiones), que sirven de ayuda especialmente a los actores de doblaje. En los siguientes ejemplos se pone letra a la risa y al grito de alegría de los personajes (la cursiva y el subrayado son míos):

(10) (RÍE)(¡Ja, ja, ja!)

(11) (GRITA)(;Yuju!) ¿Qué...?

No obstante, en este texto el traductor emplea principalmente las amplificaciones para señalar el uso de una lengua extranjera distinta del inglés, sobre todo portugués, y su traducción. En un guiño a la trama brasileña del filme, el guión original incluye intencionalmente algunos diálogos en portugués, como los siguientes (la cursiva y el subrayado son míos):

(12) (RADIO) (Portugués) "Boa noite, Rio!” (¡Buenas noches, Río!)

(13) (Portugués) "Ei, ei, você! Você aí! Vem para cá. Vamos embora. Vamos embora" (iEh, eh, tú! ¡La de ahí! Ven aquí. Vamos. Vamos).

De las 28 recurrencias, en 6 ocasiones se trata de diálogos ambientales que proceden de la radio o la televisión, como en el ejemplo 12, que buscan aclimatar al espectador a la cultura representada en el filme. No obstante, la mayoría son conversaciones directas entre personajes que buscan sorprender y confundir al espectador haciendo uso del multilingüismo (22 ocasiones): en el ejemplo 13, un regidor confunde a Linda con una de las participantes en el desfile de carnaval y comienza a darle órdenes en portugués. Al no comprender nada, la chica termina subiéndose por error a una carroza en medio de la cabalgata. Independientemente de la situación, el traductor realizó en todos los casos la misma operación: mantuvo el diálogo original, indicando previamente la lengua entre paréntesis, y ofreció la traducción en español a continuación, también entre paréntesis.

De esta forma, la decisión de marcar o no el multilingüismo como estrategia de traducción no recae directamente en el traductor, sino en los agentes posteriores, quienes valorarán en cada caso si es preferible 
mantener el portugués y que el espectador de la versión española pierda el significado de la frase, al igual que el de la versión original. O, por el contrario, se facilite su comprensión, lo que puede ocurrir con frecuencia si el destinatario directo es el público infantil, ya que, como se expone más arriba, los productos audiovisuales para niños tienden a simplificar conceptos en su traducción (O’Connell, 2003, p. 227). En este doblaje, el TA también conservó ambas opciones en la totalidad de estas recurrencias, toda vez que el TD se decantó siempre por mantener la lengua extranjera, igual que en la versión original.

\subsection{Dudas}

En el corpus sólo se han identificado tres ejemplos de duda, todas ellas referidas al multilingüismo que forma parte integrante de la película. En realidad, se trata de enunciados incomprensibles para el traductor, tales como el siguiente, pronunciado por Tulio, uno de los personajes brasileños (la cursiva y el subrayado son míos):

\section{(14) (Portugués?) “Arara! Arara! Ara-...” (GRITA)(G) (G)}

Al igual que sucede en las otras dos recurrencias encontradas, esta frase no se traduce y va precedida de la mención "(Portugués?)". El signo de interrogación nos indica que el traductor no reconoce la lengua del texto original, si bien puede intuir que es una expresión en portugués, dado el origen brasileño del personaje. De esta forma, señala esa posibilidad entre paréntesis para que los agentes sucesivos actúen en consecuencia. En la siguiente etapa, el ajustador certifica que se trata de la lengua portuguesa y suprime la marca vacilante del traductor:

TA: “¡Arara! ¡Arara! (OFF) ¡Arara!” (G) Aaaaaah ¡Uh! ¡Aaah!

TD: “¡Arara! ¡Arara! ¡Arara!” Aaaaaah ¡Uh! ¡Aaah!

\subsection{Símbolos del doblaje}

Mención aparte merecen los símbolos propios del doblaje, que el traductor de este filme añade para marcar la comunicación paralingüística o para describir el contexto en el que se presenta el diálogo. Si bien este tipo de indicación forma parte, en principio, de las tareas del ajustador, la realidad profesional de algunos estudios de doblaje demuestra que el traductor se encarga cada vez con más frecuencia de facilitar esta información (Chaume, 2012, p. 38; Cornu, 2014, p. 213). En la Tabla 5 se muestran los símbolos más recurrentes en el texto, agrupados según su significado. 
Tabla 5. Símbolos del doblaje más frecuentes

\begin{tabular}{|l|l|}
\hline Tipo de símbolo & Cantidad \\
\hline Gestos (G, GESTOS...) & 354 \\
\hline Pisa (P) & 167 \\
\hline Gritos (GRITA, GRITAN...) & 156 \\
\hline Risa (RÍE, RÍENDO...) & 138 \\
\hline Otros sonidos (BESO, RONCA, LLORA...) & 59 \\
\hline Ambiente (AD LIB, AMBIENTE...) & 26 \\
\hline Canto (CANTAN, CANTA, SILBA, TARAREA) & 29 \\
\hline Medios de comunicación (TV, RADIO) & 6 \\
\hline Otros & 7 \\
\hline TOTAL & 942 \\
\hline
\end{tabular}

En primer lugar aparecen los símbolos que describen la gesticulación paralingüística, representados como (G) o (GESTOS). En ocasiones, a este símbolo le sigue una explicitación del tipo de gesto (G ASCO) (G ERUCTO). Las indicaciones de gritos (GRITA y sus variantes), risas (RÍE y sus variantes) y otros sonidos también ocupan un lugar destacado. De esta forma, el traductor se centra casi exclusivamente en los símbolos paralingüísticos que completan la solución lingüística propuesta (768 símbolos, un $82 \%$ del total).

Por el contrario, destaca la ausencia de los símbolos que indican la procedencia de la voz (ON, OFF, DE...) y las pausas del discurso (/ o //), muy utilizados en el ámbito profesional (Marzà, Torralba y Grupo TRAMA, 2013, p.43). Estos se reducen casi exclusivamente a la (P), pisa, que aparece en 167 ocasiones (18\%) y describe el hecho de que un personaje empiece a hablar antes de que haya terminado el anterior, solapándose ambos parlamentos. En el siguiente ejemplo se ve la diferencia de simbología en el mismo fragmento del filme en el texto del traductor (TT) y el TA (la cursiva y el subrayado son míos):

TT: (TARAREA) ¡Uh! (TARAREA) ¡Ah! (TARAREA) (G) ¿Eh? Ah. Ven aquí, pajarito bonito. Oh, no pasa nada. Ya te tengo, ya está. (GESTOS).

TA: (DE) (TARAREA) ¡Uh!/(TARAREA) ¡Ah!(TARAREA)(G) ¿Eh?/ Aaah./ (DE) Anda, pajarito bonito./(ON) Oh, no tengas $\underline{(O F F)}$ miedo. Ya pasó $\underline{(O N)}$ ya pasó./ (G). 
Mientras que el traductor señala el tarareo y los gestos del personaje, el ajustador se encarga de completar el texto con los símbolos relacionados con la planificación cinematográfica y la situación del personaje en pantalla. Así, utiliza DE para avisar que el personaje está de espaldas y ON y OFF si éste aparece o no en pantalla durante la intervención. Esta información es de gran importancia para la sincronización del doblaje, ya que la escritura del texto meta se verá más o menos limitada en función de la procedencia de la voz. Si el personaje aparece de frente y en mitad de la pantalla, el respeto de la sincronía labial será más importante que si se encuentra fuera de plano. En lo que respecta al doblaje de Rio, queda patente que el ajustador es el responsable de marcar estas referencias, siendo ésta la tónica predominante en el sector del doblaje español (Marzà, Torralba y Grupo TRAMA, 2013, p. 44).

\section{Conclusiones}

Con este artículo se pretendía describir la relación dialógica existente entre los profesionales encargados del doblaje español de Rio, analizando principalmente el guión del traductor y su influencia en el texto del ajuste y el texto definitivo. Mediante la aplicación del modelo de Richart (2009, 2013) para el análisis de textos audiovisuales desde una perspectiva genética, se han identificado, clasificado y estudiado las marcas encontradas en el texto del traductor y destinadas a los siguientes agentes de la cadena de trabajo. Así, el elevado número de muestras halladas permite corroborar la primera hipótesis. Del mismo modo, también se confirma la segunda hipótesis puesto que el mantenimiento de dichas marcas depende de las restricciones audiovisuales y del destinatario del texto meta. Aunque los resultados obtenidos se ciñen únicamente al doblaje de un solo filme, y no pueden extrapolarse al resto de casos de TAV, sí pretenden servir como invitación al desarrollo de investigaciones que observen los cambios del texto traducido antes de llegar a la pantalla.

De forma paralela, se ha constatado que el traductor no es responsable de la estrategia de marcar el multilingüismo que se observa en el doblaje, puesto que su labor en las muestras analizadas se limita a indicar la presencia de la lengua extranjera y proponer una traducción acorde en forma de ampliación lingüística. Es posteriormente el ajustador y director de doblaje quien decide mantener en el texto meta las líneas de diálogo en lengua extranjera. Asimismo, en lo que concierne a los símbolos del doblaje, el traductor se encarga de señalar los símbolos paralingüísticos que complementaban la solución lingüística propuesta, dejando en manos del ajustador la tarea de escribir la mayoría de símbolos que indican la procedencia del sonido.

A la luz de estas conclusiones, resulta evidente la necesidad de recopilar, clasificar y estudiar los avant-textes de un doblaje y ser conscientes del proceso de reescritura y modelación efectuado si se quiere 
hallar a los verdaderos responsables de la traducción. Esta metodología permite asimismo revelar la transcendencia del traductor a lo largo del proceso y, paradójicamente, también la fragilidad y maleabilidad de su trabajo, características que forman parte de la naturaleza colaborativa del doblaje.

\section{Referencias}

Anderson, B., Donkin, J. C. (Productores) \& Saldanha, C. (Director). (2011). Rio [Película]. United States: Blue Sky.

Anokhina, O. (2010). De l'avant-texte à la scène: Le Molière de Boulgakov. Réflexion sur l'exogenèse. En A. Grésillon, M.-M. Mervant-Roux, \& D. Budor (Eds.), Genèses théâtrales (pp. 195-209). Paris: CNRS Éditions.

Ávila, A. (1997). El doblaje. Madrid: Cátedra.

Bellemin-Noël, J. (1972). Le texte et l'avant-texte. Paris: Larousse.

Borràs, E., \& Matamala, A. (2009). La traducció dels noms propis en pellícules d'animació infantils. Quaderns. Revista de Traducció, 16, 283-294.

Cameron, J. (Productor/Director). (1991). Terminator 2: el juicio final [Película]. United States: Carolco Pictures et al.

Chaume, F. (2004). Cine y traducción. Madrid: Cátedra.

Chaume, F. (2012). Audiovisual translation: Dubbing. Manchester: St. Jerome.

Chaume, F. (2013). Research paths in audiovisual translation: The case of dubbing. En C. Millán, \& F. Bartrina (Eds.), The Routledge Handbook of Translation Studies (pp. 288-302). New York: Routledge.

Cornu, J.-F. (2014). Le doublage et le sous-titrage. Histoire et esthétique. Rennes: Presses Universitaires de Rennes.

de Biasi, P.-M. (2011). Génétique des textes. Paris: CNRS Éditions.

Derrida, J. (1995). Mal d'archive : Une impression freudienne. Paris: Galilée.

Díaz Cintas, J. (2001). Striving for quality in subtitling: The role of a good dialogue list. En Y. Gambier \& H. Gottlieb (Eds.), (Multi) Media Translation: Concepts, Practices and Research (pp. 199-212). Amsterdam: John Benjamins.

di Carlo, C. (1995). Il doppiaggio come traduzione del film. Genesi e problemi. En S. Boujea (Ed.), Génétique et traduction (pp. 167-183). Paris: L'Harmattan.

Grésillon, A., M.-M. Mervant-Roux, \& D. Budor (2010). Introduction. En A. Grésillon, M.-M. Mervant-Roux, \& D. Budor (Eds.), Genèses théâtrales (pp. 5-23). Paris: CNRS Éditions.

Martínez Sierra, J. J. (2009). Doblar o subtitular el humor, esa no es la cuestión. The Journal of Specialized Translation, 12, 180-198.

Marzà, A., Torralba, G., \& Grupo TRAMA (2013). Las normas profesionales de la traducción para el doblaje en España. Trans, 17, 35-50.

Matamala, A. (2010). Translation for dubbing as dynamic texts: Strategies in film synchronisation. Babel, 56(2), 101-118.

Munday, J. (2013). The role of archival and manuscript research in the investigation of translator decision-making. Target, 25(1), 125-139. 
O'Connell, E. (2003). What dubbers of children's television programmes can learn from translators of children's books? Meta, 48(1-2), 222-232.

Pérez González, L. (2009). Audiovisual translation. En M. Baker \& G. Saldanha. (Eds.), Routledge encyclopedia of translation studies. Second edition (pp. 1320). New York: Routledge.

Richart, M. (2009). La alegría de transformar. Valencia: Tirant Lo Blanch.

Richart, M. (2013). Ideología y traducción. Por un análisis genético del doblaje. [Kindle version] ASIN: B00DH9JOOO. Madrid: Biblioteca nueva.

Ruzicka, V. (2009). Introducción. En V. Ruzicka (Ed.), Diálogos intertextuales 2: Bambi (pp. 7-14). Frankfurt am Main: Peter Lang.

Shavit, Z. (1980). The Ambivalent Status of Texts: The Case of Children's Literature. Poetics Today, 1(3), 75-86.

1 Los términos "proto-texto" o "inacabado" están aquí desprovistos de toda connotación negativa y sólo pretenden reflejar el constante estado de cambio al que está sometido el texto audiovisual traducido. Precisamente, la noción de "inacabado" se ha convertido en uno de los pilares teóricos de la crítica genética, como señalan Grésillon et al. (2010, p. 20).

2 Ante esta situación, desde el ámbito académico autores como Chaume (2012, p. 37) apuestan por que un solo agente -el traductor- sea el encargado de las tareas de traducción, reescritura de diálogos, segmentación en takes e inserción de símbolos.

3 Consultar Pérez González (2009) y Chaume (2013), entre otros autores, para un estado de la cuestión actualizado en investigación sobre TAV.

4 Sin ser una práctica general, las empresas propietarias del texto audiovisual incluyen cada vez con mayor frecuencia recomendaciones y sugerencias de traducción a través de guiones comentados, listas de diálogos y otros documentos anexos al guión de doblaje (Richart, 2013, epígrafe 4.1), que el traductor tiene la posibilidad de incorporar o no al texto meta (Díaz Cintas, 2001, p. 208).

5 Ficha de doblaje disponible en: http://www.eldoblaje.com/datos/FichaPelicula.asp?id=22507 [último acceso el 29/10/2015].

6 El director de doblaje de la película confirmó que la documentación proporcionada por la distribuidora al estudio de doblaje consistió en una copia del texto audiovisual y del guión original (comunicación personal). Por razones de confidencialidad, fue imposible consultar el guión original en inglés, por lo que las muestras de la versión original son fruto de una 
transcripción personal. Dado que el análisis se centra en el texto del traductor, la ausencia de guión original no afecta gravemente su contenido.

7 Las fórmulas de diálogo con los demás actores de la cadena de trabajo suelen ser unipersonales y varían de un traductor a otro. Los estudios de doblaje también siguen sus propias costumbres, lo que impide establecer unas normas profesionales en la traducción para el doblaje en España (Marzà, Torralba y Grupo TRAMA, 2013, p. 35).

8 Salvo indicación expresa, todos los ejemplos de traducción proceden del texto del traductor.

9 Según el director de doblaje Gonzalo Abril, antes de comenzar un doblaje habitualmente se llega a un acuerdo entre productora, distribuidora -marketing y departamento de doblaje-, traductor, ajustador y director de doblaje sobre las opciones de traducción de los nombres de los personajes. En este caso, hubo consenso en torno al mantenimiento del nombre original, Blu.

10 La isocronía y la sincronía labial son los dos tipos de sincronización ligados a la articulación bucal. La primera garantiza que el texto meta coincida con el tiempo que el personaje abre y cierra la boca en el parlamento original y la segunda se respeta fundamentalmente en caso de primeros planos y sobre todo si se trata de vocales abiertas (Chaume, 2012, pp. 72-75). 